\title{
Role of the coupling laser in electromagnetically induced absorption
}

\author{
Luca Spani Molella, ${ }^{*}$ Rolf-Hermann Rinkleff, ${ }^{\dagger}$ and Karsten Danzmann \\ Max-Planck-Institut für Gravitationsphysik, Albert-Einstein-Institut, and Institut für Atom- und Molekülphysik, Universität Hannover, \\ Callinstraße 38, D-30167 Hannover, Germany
}

(Received 13 May 2005; published 17 October 2005)

\begin{abstract}
The cesium $D_{2}$ line closed hyperfine transition $F=4 \rightarrow F^{\prime}=5$ was simultaneously coupled and probed in a hot atomic beam. We experimentally showed that, when the probe laser frequency approached that of the two-photon-resonance corresponding to electromagnetically induced absorption conditions, an enhanced transparency of the coupling laser appeared, combined with a further central absorption peak at zero probe laser detuning. Simultaneously, the coupling laser parametric dispersion showed a broad negative pattern together with a narrower steep positive phase shift in correspondence with the two-photon resonance, with sub-Doppler half-widths down to about $10 \mathrm{kHz}$.
\end{abstract}

DOI: 10.1103/PhysRevA.72.041802

\section{INTRODUCTION}

Since the discovery of electromagnetically induced absorption (EIA [1]) much interest has been devoted to measurements [2], numerical simulations, and modeling $[3,4]$ of this phenomenon, a complementary effect to electromagnetically induced transparency (EIT [5-7]). Even though many absorption and several dispersion measurements of EIA have been performed in alkali atoms [8,9], no experimental attention has been dedicated to the behavior of the coupling laser, neither regarding its absorption nor its dispersion. Our heterodyne interferometer allows simultaneous absorption and dispersion measurements of either the probe or the coupling laser. It is, therefore, well suited to perform the lacking measurements and complete the experimental description of this transition. Moreover, the use of a cesium atomic beam eliminates unwanted Doppler broadening of the lines.

\section{SYSTEM}

We studied EIA in a closed two level system of the ${ }^{133} \mathrm{Cs}$ $D_{2}$ line $(852 \mathrm{~nm})$, which was coupled and probed with two linearly polarized lasers orthogonally polarized to each other. A theoretical explanation of EIA is given in [10] and extended, together with additional numerical calculations for rubidium, in [4]. The transition under investigation involved the hyperfine levels $F=4$ and $F^{\prime}=5$ (Fig. 1).

In this degenerate $\Lambda$ system, the ground levels coincided for the coupling and the probe laser.

In cesium the hyperfine splitting between the $F$ hyperfine sublevels amounts to roughly $9.2 \mathrm{GHz}$ while the hyperfine splitting of the $F^{\prime}$ sublevels is of the order of $150-250 \mathrm{MHz}$ depending on the sublevels considered. Since the magnetic field was compensated, the Zeeman splitting of the levels could be ignored.

According to Lezama et al. [11] the appearance of EIA needs to satisfy the following conditions (that can even be relaxed as pointed out in [3]): (1) the transition between the ground and the excited level must be closed; (2) the ground

\footnotetext{
*Electronic address: Luca.Spani.Molella@aei.mpg.de

${ }^{\dagger}$ Electronic address: Rolf-Hermann.Rinkleff@aei.mpg.de
}

PACS number(s): 42.50.Gy, 42.62.Fi, 32.70.Jz

level must be degenerate (i.e., the condition $F>0$ must be fulfilled); (3) the total angular momentum of the excited level $F^{\prime}$ must be greater than the angular momentum of the ground level (i.e., $F^{\prime}>F$ ). All three of these conditions were satisfied by our system.

If condition (3) is not fulfilled, the system behaves like a usual $\Lambda$ system, generating an EIT signal. We were able to verify this with the same setup, locking the coupling and the probe laser to the $F=3 \rightarrow F^{\prime}=2$ transition and obtaining the expected behavior.

\section{SETUP}

The measurements were conducted with a heterodyne interferometer. This apparatus was already used to study the parametric dispersion in electromagnetically induced transparency $[12,13]$. The apparatus was modified to reduce possible interferences between the optical paths and to improve the stability of the phase-locked loops. In particular, we designed a new optical path for the reference arm, as shown in Fig. 2.

The heterodyne interferometer was realized using three single-mode diode lasers (in the following called coupling,

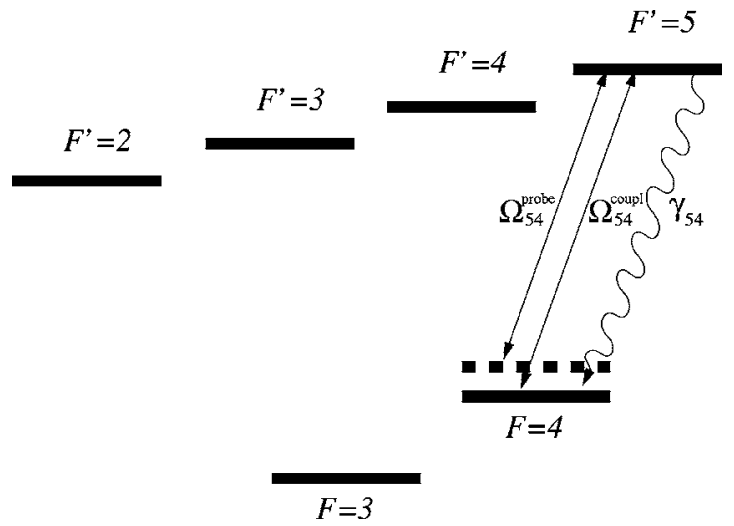

FIG. 1. Level schematic of the $D_{2}$ line of atomic Cs. The $F$ levels are the hyperfine levels of the ground level $6 s^{2} S_{1 / 2}$. The $F^{\prime}$ are the hyperfine levels of the excited level $6 p^{2} P_{3 / 2} \cdot \gamma_{54}$, spontaneous emission rates; $\Omega_{54}$, Rabi frequencies for the considered transition 


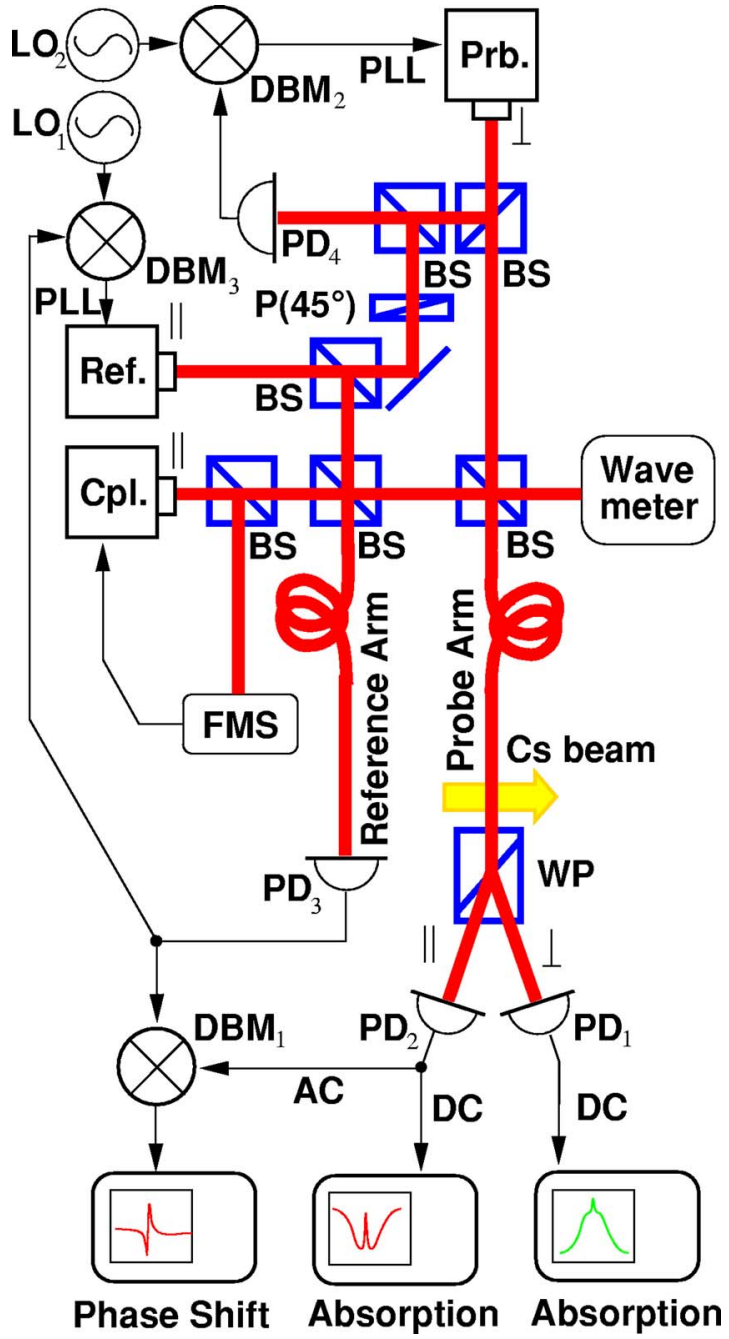

FIG. 2. (Color online) Experimental setup. Cpl., Ref., Prb.: coupling, reference, and probe laser; $\mathrm{P}\left(45^{\circ}\right)$ : polarizer under $45^{\circ}$ with respect to incident polarization; WP: Wollastone prism; PD: photodiode; LO: local oscillator; PLL: optical phase-locked loop; DBM: double balanced mixer; BS: power beam splitter; FMS: frequency modulation spectroscopy.

probe, and reference laser). The outcoming light was linearly polarized; the coupling and reference laser shared the same polarization: perpendicular to that of the probe laser. We used self-injection locked lasers, passively stabilized with an external optical feedback [14]. To fix an absolute value for the frequency and to avoid long time scale drifts, the coupling laser was actively locked to the transition between the levels $6 S_{1 / 2}, F=4$ and $6 P_{3 / 2}, F^{\prime}=5$ using frequency modulation spectroscopy.

Our experiment required a strong phase correlation between all the lasers involved; for this reason, the three lasers were connected in a chain of two subsequent phase-locked loops. The reference laser was locked to the coupling laser (via the local oscillator $\mathrm{LO}_{1}$ ) with a fixed offset $\Delta \nu_{\text {off }}$ $\approx 1 \mathrm{GHz}$; the probe laser was locked to the reference laser with a tunable offset $\Delta \nu \approx \delta+1 \mathrm{GHz}$ (via the local oscillator $\left.\mathrm{LO}_{2}\right)$. The offsets $\Delta \nu_{\text {off }}$ and $\Delta \nu$ had opposite signs, so that the residual offset between the coupling and probe laser amounted to exactly $\delta$, which was the independent variable during data taking. The varying of $\delta$ was computer controlled through the local oscillator $\mathrm{LO}_{2}$. During each measurement, lasting approximately $2 \mathrm{~min}$, about 10000 data points were acquired.

In our experiment the laser fields coming from the three laser sources were superimposed well before the interaction zone. This minimized the relative phase noise between the three lasers due to mechanical and acoustical perturbations of the optical setup. Before passing through the atomic beam (in the probe arm) or reaching the photodiode (in the other arm) all the lasers were guided through single-mode fibers, allowing us to achieve extremely good alignment and mode matching between the beams, which was accompanied by a loss in power of about 50\%. Polarizers and half-wave plates at the laser outputs (not shown in Fig. 2) enabled us to obtain the desired polarization adjustment at the interaction zone.

The detuning of the reference laser from the resonance frequencies was about $1 \mathrm{GHz}$, so that no interaction with the system could be assumed. This frequency gap was indeed too large to stimulate a hyperfine transition from the level $F=4$ to any $F^{\prime}$ excited level and too small to stimulate a hyperfine transition from the level $F=3$.

After the interaction zone, a Wollastone prism separated the two different light polarizations. Since the coupling and reference laser shared the same polarization, they produced a beat at the same photodiode $\left(\mathrm{PD}_{2}\right)$. Light coming from the coupling and the reference laser and not interacting with the medium was also present in the reference arm of the interferometer and generated a beat at the photodiode $\mathrm{PD}_{3}$. Mixing the RF-beat note signals from $\mathrm{PD}_{2}$ and $\mathrm{PD}_{3}$, provided the information about the phase shift of the coupling laser caused by its interaction with the probe laser and the medium.

Calling $d \varphi(\delta)$ the phase shift induced by the variation of the refractive index in the proximity of the resonance, $\alpha_{\mathrm{c}}$ the absorption coefficient for the coupling laser, and $E_{0}^{j}$ the electrical field of the $j$ th laser, the input signal at the double balanced mixer $\mathrm{DBM}_{1}$ was:

$$
S_{\text {mix }}(\delta) \propto\left(E_{0}^{\mathrm{c}} E_{0}^{\mathrm{r}}\right)^{2} e^{-\alpha_{\mathrm{c}}(\delta) l} \sin [d \varphi(\delta)-\phi],
$$

where $\phi=2 \pi \Delta \nu_{\text {off }}\left(L_{\text {probe }}-L_{\text {ref }}\right) / c$ could be set to a multiple of $2 \pi$ by an appropriate choice either of the reference laser frequency (which was done here) or of the arm lengths $L_{\text {probe }}$ and $L_{\text {ref }}$. The probe and coupling beams absorption signals could be extracted from the intensity of the light impinging on the photodiodes $\mathrm{PD}_{1}$ and $\mathrm{PD}_{2}$. As the reference laser was not interacting with the medium its power contributed only with a constant offset. Since this power remained unchanged during a whole measurement set, it could be easily measured and subtracted at the end of the session. If $I_{i}$ was the intensity at the $i$ th photodiode, one had $I_{1}(\delta) \propto\left(E_{0}^{\mathrm{p}}\right)^{2} e^{-2 \alpha_{\mathrm{p}}(\delta) l}$ and $I_{2}(\delta) \propto\left(E_{0}^{\mathrm{c}}\right)^{2} e^{-2 \alpha_{\mathrm{c}}(\delta) l}+\left(E_{0}^{\mathrm{r}}\right)^{2}$, calling $\alpha_{\mathrm{c}}$ and $\alpha_{\mathrm{p}}$ the absorption coefficients for the coupling and the probe laser.

The sample consisted of an atomic beam crossing the probe arm orthogonally to the laser beams. It was produced by a continuous flow reflux oven at about $500 \mathrm{~K}$ with a collimation ratio of 1:50. The residual Doppler width of the 


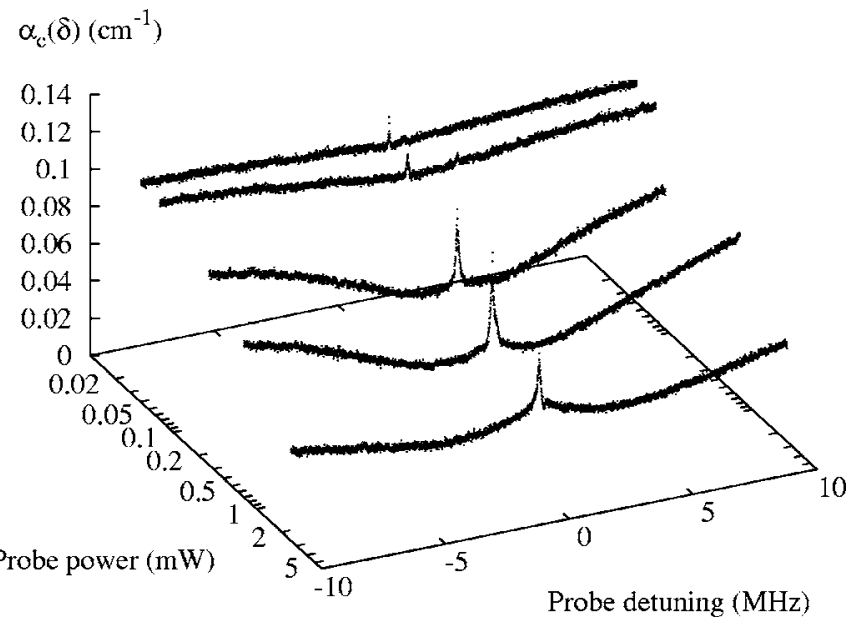

FIG. 3. Coupling laser absorption coefficient for probe laser powers of $2.60 \mathrm{~mW}, 0.71 \mathrm{~mW}, 0.27 \mathrm{~mW}, 69 \mu \mathrm{W}$, and $41 \mu \mathrm{W}$, respectively and a constant coupling laser power of $0.17 \mathrm{~mW}$.

optical transition was 4.5 MHz. The laser beams at the interaction zone had a waist of $2.2 \mathrm{~mm}$ while the atomic beam width was $5 \mathrm{~mm}$. Six Helmoltz coils in the three spatial directions suppressed unwanted magnetic fields in the interaction region below $0.5 \mu \mathrm{T}$.

\section{EXPERIMENTAL RESULTS}

During each measurement the probe laser detuning was swept over a $20 \mathrm{MHz}$ interval. Figure 3 shows the coupling laser absorption coefficient at five different probe laser powers and a constant coupling power of $0.17 \mathrm{~mW}$.

When the probe laser frequency approached that of the two-photon resonance the absorption coefficient was reduced. However, at the two-photon resonance, an increase in the parametric absorption was observed. The overall transparency effect increased for increasing probe laser power. At the two-photon resonance its amplitude was almost compensated by the central absorption peak.

Goren et al. $[4,15]$ predicted, for a similar configuration in rubidium, a decrease in the central absorption peak for high coupling intensities (i.e., higher coupling to probe power ratios). Assuming that the change in amplitude was not covered by the detection noise (which could be suppressed down to about $1 \%$ of the signal), such an effect was not observed even for coupling laser power of about $8 \mathrm{~mW}$ and coupling to probe power ratios greater than about 100 .

At low probe laser powers (i.e. high coupling to probe power ratios) both effects tended to disappear. The coupling laser approached the constant absorption level of the coupled transition keeping its structure of a broad transparency in the vicinity of the two-photon resonance with a central absorption peak at zero probe detuning. Typical parametric absorption and dispersion measurements for the coupling laser are shown in Fig. 4.

To investigate the dependence of the phase shift on the laser powers, we fitted the different dispersion curves obtained at various powers to a phenomenological dispersion curve, thus obtaining information on their width and steep-
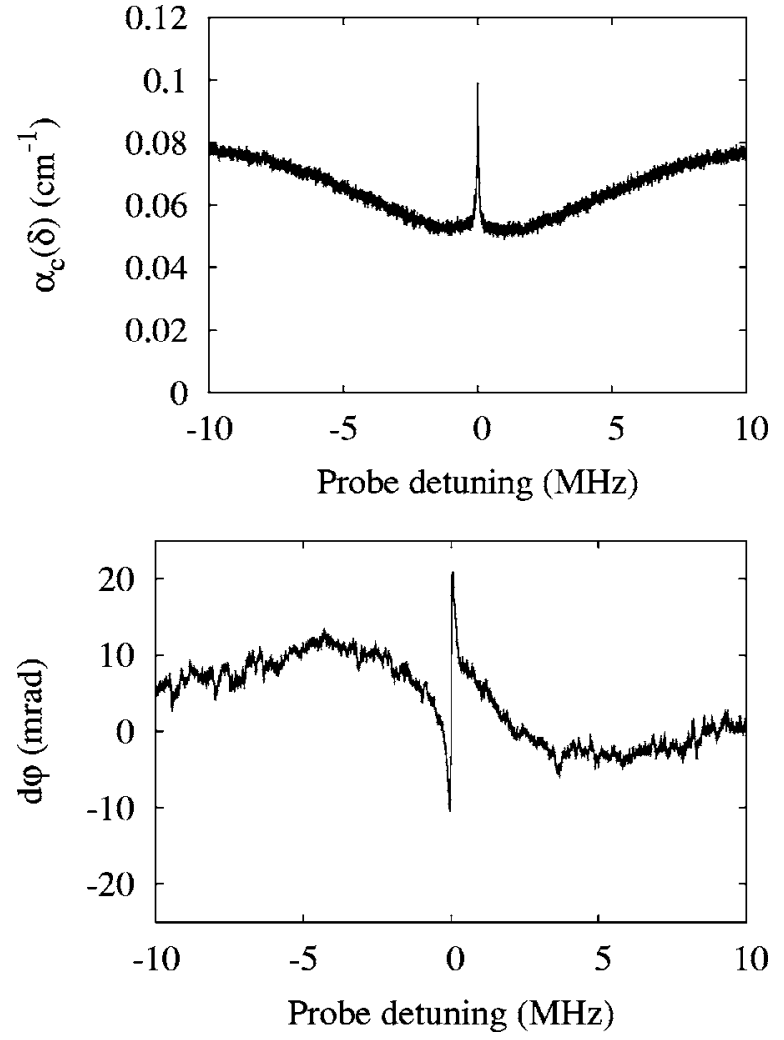

FIG. 4. Typical coupling laser absorption coefficient (above) and phase shift (below) corresponding to a probe laser EIA transition. The coupling and probe laser power are $0.16 \mathrm{~mW}$ and $0.25 \mathrm{~mW}$ (above) and $16 \mu \mathrm{W}$ and $0.16 \mathrm{~mW}$ (below).

ness. At first (Fig. 5) we kept the probe laser at $0.26 \mathrm{~mW}$ using different coupling laser powers (from $1.4 \mu \mathrm{W}$ to $0.18 \mathrm{~mW}$ ). In a second experiment (Fig. 6) we varied the probe field power from $25 \mu \mathrm{W}$ to $3.2 \mathrm{~mW}$ while keeping the coupling power constant $(0.16 \mathrm{~mW})$. We repeated this last measurement on two different days under identical experimental conditions. The coupling laser parametric dispersion (Fig. 6) showed a maximum as a function of the probe laser

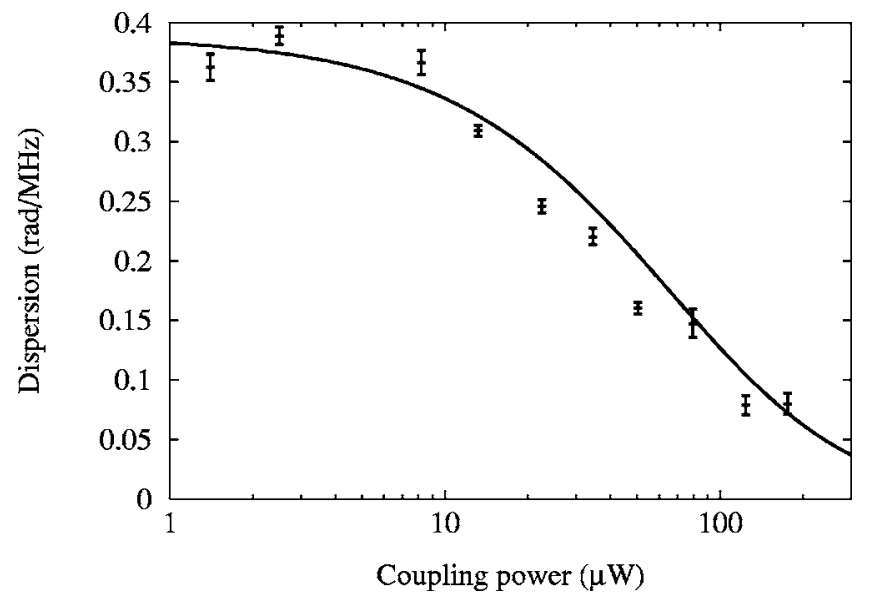

FIG. 5. Absolute values of the observed coupling field dispersion at $0.26 \mathrm{~mW}$ probe laser power. The curve simulates the system under comparable experimental conditions. 


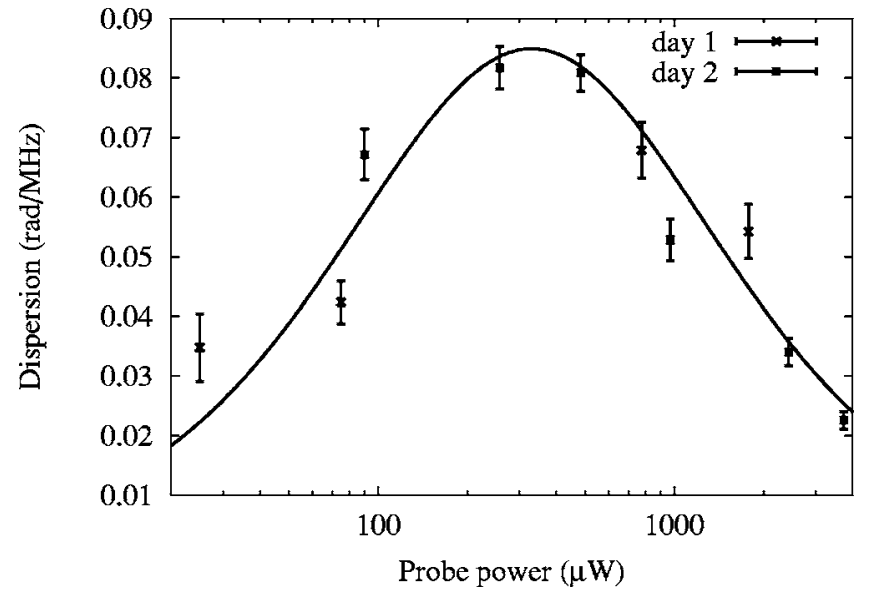

FIG. 6. Absolute values of the observed coupling field dispersion at $0.16 \mathrm{~mW}$ coupling laser power. The curve simulates the system under comparable experimental conditions.

power. Conversely, when the coupling laser power was changed, we observed (Fig. 5) a decrease in the dispersion value with increasing power. The error bars represent the deviation of the measured curves from the phenomenological patterns. The solid lines are the result of a simulation of the system (following the model of [13]) under the same experimental conditions (interaction length, laser beam section, atomic density, Rabi frequencies, spontaneous decay rate, etc.) and well reproduce the pattern of the experimental points. In contradistinction to [4], no transition from positive to negative dispersion was found, even in the power range where no transparency was observed. This can be explained considering that the coupling absorption approached a quasiconstant level keeping unchanged its qualitative structure as a function of the probe detuning.

We measured signals with a half-width at half maximum starting from about $10 \mathrm{kHz}$ and remaining under $100 \mathrm{kHz}$ for various laser powers. Figure 7 shows the width of the dispersion curve when keeping the coupling laser power fixed at $16 \mu \mathrm{W}$. The observed half-widths were well below the halfwidths of parametric dispersion curves observed in $\Lambda$ systems in Cs. A similar result for the regular probe absorption spectra in the EIA and EIT configurations has been discussed in $[8]$.

[1] A. M. Akulshin, S. Barreiro, and A. Lezama, Phys. Rev. A 57, 2996 (1998).

[2] A. M. Akulshin, A. Cimmino, A. I. Sidorov, P. Hannaford, and G. I. Opat, Phys. Rev. A 67, 011801(R) (2003).

[3] C. Goren, A. D. Wilson-Gordon, M. Rosenbluh, and H. Friedmann, Phys. Rev. A 67, 033807 (2003).

[4] C. Goren, A. D. Wilson-Gordon, M. Rosenbluh, and H. Friedmann, Phys. Rev. A 69, 053818 (2004).

[5] G. Alzetta, A. Gozzini, L. Moi, and G. Orriols, Nuovo Cimento Soc. Ital. Fis., B 36B, 5 (1976).

[6] S. E. Harris, Phys. Today 50(7), 36 (1997).

[7] E. Arimondo, in Progress in Optics, edited by E. Wolf (Elsevier Science, Amsterdam, 1996), Vol. 35, pp. 257-354.

[8] A. M. Akulshin, A. I. Sidorov, R. J. McLean, and P. Hannaford, J. Opt. B: Quantum Semiclassical Opt. 6, 491

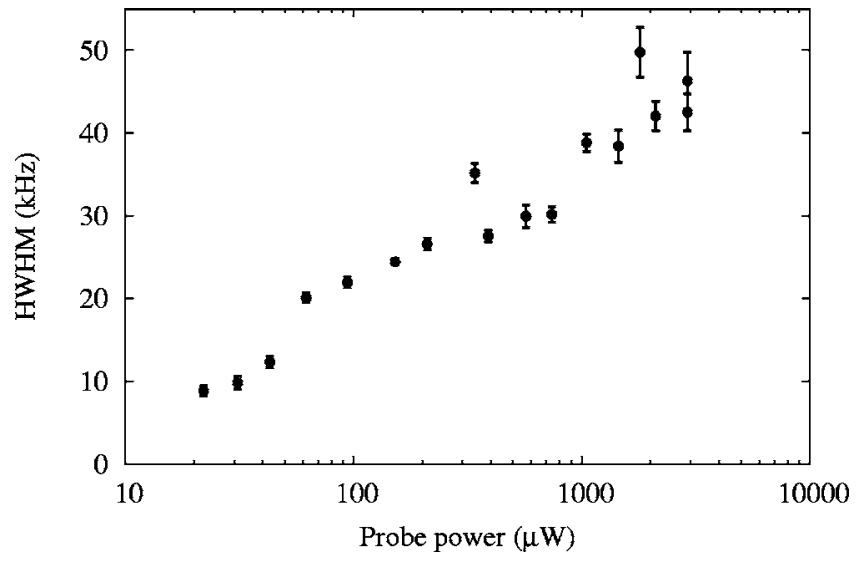

FIG. 7. Linewidth of the coupling laser parametric dispersion as a function of the probe field power at a constant coupling power of $16 \mu \mathrm{W}$. HWHM: half-width at half maximum.

The measurements were limited by the diode laser powers at high power and by the detection noise and the stability of the phase-locked loops at low power.

\section{CONCLUSIONS}

For a closed system exhibiting EIA on the probe laser, we have shown that the coupling laser experiences a broad parametric transparency with a central absorption peak for various probe laser powers. The absorptive characteristics of the system indicate that the coupling and the probe laser play two different roles in the phenomenon (as opposed to what happens in $\Lambda$ systems, where both show EIT). In this system the measured parametric linewidths for the coupling field spectra are comparable to the two-photon linewidths typical for the regular EIA probe field spectra, but remain under $100 \mathrm{kHz}$ over a much wider probe power range.

\section{ACKNOWLEDGMENTS}

We thank the Deutsche Forschungsgemeinschaft for its support through the Grant No. SFB407, and Professor A. Wilson-Gordon and Dr. C. Goren for fruitful discussions.

(2004).

[9] A. M. Akulshin, S. Barreiro, and A. Lezama, Phys. Rev. Lett. 83, 4277 (1999).

[10] A. V. Taichenachev, A. M. Tumaikin, and V. I. Yudin, Phys. Rev. A 61, 011802(R) (1999).

[11] A. Lezama, S. Barreiro, and A. M. Akulshin, Phys. Rev. A 59, 4732 (1999).

[12] M. Müller, F. Homann, R.-H. Rinkleff, A. Wicht, and K. Danzmann, Phys. Rev. A 62, 060501(R) (2000).

[13] M. Müller, F. Homann, R.-H. Rinkleff, A. Wicht, and K. Danzmann, Phys. Rev. A 64, 013803 (2001).

[14] B. Dahmani, L. Hollberg, and R. Drullinger, Opt. Lett. 12, 876 (1987).

[15] C. Goren (private communication). 